\title{
A REMARK ON OPTIMAL VARIANCE STOPPING PROBLEMS
}

\author{
BRUNO BUONAGUIDI, ${ }^{*}$ Bocconi University
}

\begin{abstract}
In an optimal variance stopping problem the goal is to determine the stopping time at which the variance of a sequentially observed stochastic process is maximized. A solution method for such a problem has been recently provided by Pedersen (2011). Using the methodology developed by Pedersen and Peskir (2012), our aim is to show that the solution to the initial problem can be equivalently obtained by constraining the variance stopping problem to the expected size of the stopped process and then by maximizing the solution to the latter problem over all the admissible constraints. An application to a diffusion process used for modeling the dynamics of interest rates illustrates the proposed technique.
\end{abstract}

Keywords: Constrained and unconstrained variance optimal stopping problems; diffusion process; interest rate; nonlinear optimal stopping problem

2010 Mathematics Subject Classification: Primary 60G40

Secondary $62 \mathrm{~L} 15 ; 90 \mathrm{C} 20$

\section{Introduction}

Let $X=\left(X_{t}\right)_{t \geq 0}$ be a continuous-time real-valued Markov process and let $\mathbb{P}_{x}$ be the probability measure under which $X$ starts at $x$. Let us define

$$
\mathbb{V}(x):=\sup _{\tau} \operatorname{var}_{x}\left[X_{\tau}\right]
$$

where the supremum is taken over all the stopping times $\tau$ of $X$ such that $\mathbb{E}_{x}\left[X_{\tau}^{2}\right]<\infty$. The optimal variance stopping problem for $X$ aims at computing the value function $\mathbb{V}(x)$ and determining the optimal stopping time $\tau^{\star}(x)$ at which the supremum on the right-hand side of (1.1) is attained. Recalling that $\operatorname{var}_{x}\left[X_{\tau}\right]:=\mathbb{E}_{x}\left[\left(X_{\tau}-\mathbb{E}_{x}\left[X_{\tau}\right]\right)^{2}\right]=\mathbb{E}_{x}\left[X_{\tau}^{2}\right]-\left(\mathbb{E}_{x}\left[X_{\tau}\right]\right)^{2}$, we note that the expectation operator of the stopped process enters the value function (1.1) in a nonlinear (quadratic) way, unlike what happens in a standard-linear optimal stopping problem. This implies that the well established theory of optimal stopping (see, e.g. Peskir and Shiryaev (2006) or Shiryaev (1978)) does not directly work for (1.1).

An effective method for solving an optimal variance stopping problem has been recently proposed by Pedersen (2011). His idea relies on embedding (1.1) into the linear optimal stopping problem

$$
W_{c}(x):=\sup _{\tau} \mathbb{E}_{x}\left[\left(X_{\tau}-c\right)^{2}\right], \quad c \in \mathbb{R},
$$

for which the standard optimal stopping theory can be applied. He proved that if there exists a constant, say $c(x)$, such that $W_{c(x)}(x)$ is finite and the associated optimal stopping time $\tau_{c(x)}$

Received 23 May 2014; revision received 29 November 2014.

* Postal address: Department of Decision Sciences, Bocconi University, Via Roentgen 1, 20136 Milan, Italy.

Email address: bruno.buonaguidi@phd.unibocconi.it 
satisfies

$$
c(x)=\mathbb{E}_{x}\left[X_{\tau_{c(x)}}\right],
$$

then $\tau^{\star}(x)=\tau_{c(x)}$ and $\mathbb{V}(x)=W_{c(x)}(x)$.

In this paper we provide an alternative method for tackling an optimal variance stopping problem; our approach is based on the technique originally introduced and developed by Pedersen and Peskir (2012). In particular, in the next section we show that the solution to (1.1) can be obtained by first solving a constrained optimal stopping problem, where the constraint is given on the expectation of the stopped process, and then by maximizing the obtained solution over the set of all the admissible constraints. By exploiting this result, in Section 3 we derive the explicit solution to (1.1), under the assumption that $X$ follows the diffusion process used by Conley et al. (1997) and Ahn and Gao (1999) for modeling the dynamics of interest rates. Section 4 concludes with a summary discussion. The proofs of the results contained in Sections 2 and 3 follow closely the proof of Theorem 3 in Pedersen and Peskir (2012).

\section{Main result}

In this section we state and prove the main result of this paper. In the what follows, we denote by $\mathcal{C}_{x}$ the range of the map $\tau \mapsto \mathbb{E}_{x}\left[X_{\tau}\right]$, where $\tau$ is a stopping time of the Markov process $X=\left(X_{t}\right)_{t \geq 0}$, starting at $x$ under $\mathbb{P}_{x}$.

Theorem 2.1. For a given constant $\lambda$, consider the optimal stopping problem

$$
U_{\lambda}(x):=\sup _{\tau} \mathbb{E}_{x}\left[X_{\tau}^{2}-\lambda X_{\tau}\right]
$$

where the supremum is taken over all the stopping times of $X$ such that $\mathbb{E}_{x}\left[X_{\tau}^{2}\right]<\infty$. Let $\tau_{\lambda}^{U}$ be its optimal stopping time and $m \in \mathcal{C}_{x}$. If there exists a constant $\lambda(m, x)$ such that

$$
m=\mathbb{E}_{x}\left[X_{\tau_{\lambda(m, x)}^{U}}\right]
$$

then $\tau_{m}^{V}(x):=\tau_{\lambda(m, x)}^{U}$ is an optimal stopping time for the constrained optimal stopping problem

$$
V_{m}(x):=\sup _{\tau: \mathbb{E}_{x}\left[X_{\tau}\right]=m} \mathbb{E}_{x}\left[X_{\tau}^{2}\right]
$$

The value function $\mathbb{V}(x)$ from (1.1) is given by

$$
\mathbb{V}(x)=\sup _{m \in \mathcal{C}_{x}}\left(V_{m}(x)-m^{2}\right)
$$

and the associated optimal stopping time $\tau^{\star}(x)$ can be expressed as $\tau_{\lambda\left(m^{\star}, x\right)}^{U}=\tau_{m^{\star}}^{V}(x)$, where $m^{\star}$ is the value at which the supremum in (2.4) is attained.

Proof. We can write (1.1) by conditioning on the magnitude $m \in \mathcal{C}_{x}$ of $\mathbb{E}_{x}\left[X_{\tau}\right]$ :

$$
\begin{aligned}
\mathbb{V}(x) & =\sup _{\tau}\left(\mathbb{E}_{x}\left[X_{\tau}^{2}\right]-\left(\mathbb{E}_{x}\left[X_{\tau}\right]\right)^{2}\right) \\
& =\sup _{m \in \mathcal{C}_{x}} \sup _{\tau: \mathbb{E}_{x}\left[X_{\tau}\right]=m}\left(\mathbb{E}_{x}\left[X_{\tau}^{2}\right]-\left(\mathbb{E}_{x}\left[X_{\tau}\right]\right)^{2}\right) \\
& =\sup _{m \in \mathcal{C}_{x}}\left(V_{m}(x)-m^{2}\right),
\end{aligned}
$$


which establishes (2.4) and makes evident that (1.1) requires solving (2.3). To this aim let us define the Lagrange function

$$
L_{x, m}(\tau, \lambda):=\mathbb{E}_{x}\left[X_{\tau}^{2}\right]-\lambda\left(\mathbb{E}_{x}\left[X_{\tau}\right]-m\right)
$$

for $x$ and $m \in \mathcal{C}_{x}$ given and fixed. It is easy to observe that the stopping time $\tau_{\lambda}^{U}$, optimal in (2.1), realizes

$$
L_{x, m}\left(\tau_{\lambda}^{U}, \lambda\right)=\sup _{\tau} L_{x, m}(\tau, \lambda)
$$

It follows that if there exists a constant $\lambda(m, x)$ so that $\tau_{\lambda(m, x)}^{U}$ satisfies (2.1) and (2.2), then from (2.6) and (2.7), we have

$$
\mathbb{E}_{x}\left[X_{\tau_{\lambda(m, x)}^{U}}^{2}\right]=L_{x, m}\left(\tau_{\lambda(m, x)}^{U}, \lambda\right) \geq \mathbb{E}_{x}\left[X_{\tau}^{2}\right]
$$

for any stopping time $\tau$ such that $\mathbb{E}_{x}\left[X_{\tau}\right]=m$. This shows that the stopping time $\tau_{m}^{V}(x)$, optimal in (2.3), coincides with $\tau_{\lambda(m, x)}^{U}$ and, together with (2.5), also proves that $\tau^{\star}(x)=$ $\tau_{\lambda\left(m^{\star}, x\right)}^{U}=\tau_{m^{\star}}^{V}(x)$.

It is straightforward to note that the expression $V_{m}(x)-m^{2}$ appearing in (2.4) equals the value function $\mathbb{V}(x)$ subject to $\mathbb{E}_{x}\left[X_{\tau}\right]=m$, with $m \in \mathcal{C}_{x}$. This means that $\mathbb{V}(x)$ can be obtained by maximizing a constrained optimal variance stopping problem over the set of all the admissible constraints. The idea of solving such a constrained optimal stopping problem is already present in Pedersen (2011, Remark 3.3), but there is no connection with its unconstrained version. Hence, Theorem 2.1 establishes a bridge between constrained and unconstrained optimal variance stopping problems: the approach used therein relies on the very powerful methodology developed by Pedersen and Peskir (2012) in order to solve mean-variance optimal stopping problems.

\section{Financial application}

The variance is one of the main criteria adopted in finance for quantifying the risk of a market variable. Usually, the higher its variance, the more risky it is perceived. Problem (1.1) is therefore relevant when one is sequentially monitoring a market variable $X=\left(X_{t}\right)_{t \geq 0}$ and aims at detecting the moment at which its variance attains the maximum value: at that time, hedging or speculation strategies can be realized.

In this section we solve the optimal variance stopping problem (1.1) for the diffusion process $X$, governed under $\mathbb{P}_{x}$ by the following stochastic differential equation:

$$
\mathrm{d} X_{t}=-k X_{t}^{2} \mathrm{~d} t+\sigma X_{t}^{3 / 2} \mathrm{~d} B_{t}, \quad X_{0}=x>0 .
$$

In the above expression we denote by $\mu(z):=-k z^{2}$ and $\sigma(z):=\sigma z^{3 / 2}$ the drift and diffusion term of $X$, respectively, and we set $k, \sigma>0$. This process is used for modeling the dynamics of interest rates and was introduced by Conley et al. (1997) and Ahn and Gao (1999), in order to capture the results of some empirical studies, which had showed that the evolution of interest rates is characterized by a nonlinear drift and a diffusion term proportional to $x^{3 / 2}$; see, e.g. Aiit-Sahalia (1996). The state space of this process is $(0, \infty)$. Furthermore, we assume that $2 k>\sigma^{2}$, so that

$$
2 x \mu(x)+\sigma^{2}(x) \leq C\left(1+x^{2}\right)
$$


holds for any $x>0$ and a nonnegative constant $C$. Condition (3.2) ensures the existence of a strong solution to (3.1); see, e.g. Kutoyants (2004) and Karatzas and Shreve (1988, Chapter 5) for the concept of a strong solution.

Let us analyze some properties of $X$. Its scale function $S(x)$ and speed measure $m(\mathrm{~d} x)$ are given by

$$
\begin{gathered}
S(x):=\int^{x} \exp \left(-\int^{y} \frac{2 \mu(z)}{\sigma^{2}(z)} \mathrm{d} z\right) \mathrm{d} y=\frac{x^{1+\gamma}}{1+\gamma} \\
m(\mathrm{~d} x):=\frac{1}{\sigma^{2}(x) S^{\prime}(x)}=\frac{1}{\sigma^{2} x^{3+\gamma}},
\end{gathered}
$$

where $\gamma:=2 k / \sigma^{2}>1$; then, it is easy to verify that

$$
\begin{array}{cc}
S(0+)=0, & S(\infty-)=\infty, \\
\int_{0+}^{\varepsilon} m(\mathrm{~d} x)=\infty, & \int_{0+}^{\varepsilon} S(x) m(\mathrm{~d} x)=\infty,
\end{array}
$$

being $\varepsilon$ any arbitrary point greater than 0 . Denoted by $\tau_{y}:=\inf \left\{t \geq 0: X_{t}=y\right\}$ the first hitting time to $y$ and $\tau_{0+}:=\lim _{y \downarrow} \tau_{y}$, from (3.4) we read that 0 is an attracting boundary, meaning that $\mathbb{P}_{x}\left(\tau_{0+} \leq \tau_{b}\right)>0$ for all $x$ and $b$ such that $0<x<b$, and $X$ is transient, namely, $X_{t} \rightarrow 0$ $\mathbb{P}_{x}$-almost surely (a.s.) as $t \rightarrow \infty$. From (3.5) we read that 0 is a natural boundary for $X$, in the sense that $X$ can neither start from it nor attain it in finite mean time; see, e.g. Karlin and Taylor (1981, Chapter 15). Moreover, from the general theory of diffusion processes and (3.3), we have

$$
\mathbb{P}_{x}\left(\tau_{b}<\tau_{0+}\right)=\frac{S(x)-S(0+)}{S(b)-S(0+)}=\left(\frac{x}{b}\right)^{1+\gamma}, \quad 0<x<b .
$$

The conditional distribution of $X_{t}, t \geq 0$, is well known (see Ahn and Gao (1999)); in particular its variance is given by

$$
\operatorname{var}_{x}\left[X_{t}\right]=\frac{\alpha^{2}(t) \mathrm{e}^{-u(t, x)}}{q}\left(\frac{M(q-1,1+q, u(t, x))}{q-1}-\frac{\mathrm{e}^{-u(t, x)}(M(q, 1+q, u(t, x)))^{2}}{q}\right),
$$

where $\alpha(t):=2 /\left(\sigma^{2} t\right), u(t, x):=\alpha(t) / x, q:=2\left(k+\sigma^{2}\right) / \sigma^{2}-1$, and $M(a, b, x)$ is the Kummer confluent hypergeometric function defined by

$$
M(a, b, x):=1+\frac{a}{b} x+\frac{a(a+1)}{b(b+1)} \frac{x^{2}}{2 !}+\cdots .
$$

From (3.7) and (3.8) we observe that $\operatorname{var}_{x}\left[X_{t}\right] \rightarrow 0$ as $t \rightarrow \infty$, which suggests that the value function (1.1) could be finite.

Following the lines of Theorem 2.1, we begin by deriving the solution to the optimal stopping problem (2.1). The result is shown in the next proposition.

Proposition 3.1. Let $X$ be the diffusion process satisfying (3.1) and (3.2) and let $\gamma:=2 \mathrm{k} / \sigma^{2}>$ 1. Then, for a given constant $\lambda>0$, the function $U_{\lambda}(x)$ in (2.1) is given by

$$
U_{\lambda}(x)= \begin{cases}{\left[(b(\lambda))^{1-\gamma}-\lambda(b(\lambda))^{-\gamma}\right] x^{1+\gamma},} & 0<x \leq b(\lambda) \\ x^{2}-\lambda x, & x \geq b(\lambda)\end{cases}
$$


where $b(\lambda):=\lambda \gamma /(\gamma-1)$. The stopping time

$$
\tau_{b(\lambda)}^{+}:=\inf \left\{t \geq 0: X_{t} \geq b(\lambda)\right\}
$$

is optimal in (2.1), that is, $\tau_{\lambda}^{U}=\tau_{b(\lambda)}^{+}$.

Proof. Denote by $G_{\lambda}(x):=x^{2}-\lambda x, x>0$, the gain function in (2.1). It is immediate to note that $x \mapsto G_{\lambda}(x)$ is convex on $(0, \infty)$, satisfies $G_{\lambda}(0)=G_{\lambda}(\lambda)=0, G_{\lambda}(+\infty)=+\infty$, and $G_{\lambda}(\lambda / 2)=-\lambda^{2} / 4$, being $\lambda / 2$ its unique global minimum. We also know that $X_{t} \rightarrow 0$ $\mathbb{P}_{x}$-a.s. as $t \rightarrow \infty$. Hence, we conjecture that there exists a threshold $b \geq \lambda$, such that the optimal stopping time $\tau_{\lambda}^{U}$ in (2.1) equals $\tau_{b}^{+}$, where $\tau_{b}^{+}$is the first time that $X$ is above $b$ and is defined through (3.10) above. We recall that the infinitesimal generator $\mathbb{L}$ of $X$ acts on $f \in C^{2}((0, \infty))$ as $(\mathbb{L} f)(x):=\left(\sigma^{2} / 2\right) x^{3} f^{\prime \prime}(x)-k x^{2} f^{\prime}(x)$.

The strong Markov property of $X$ and the theory of optimal stopping (see, e.g. Peskir and Shiryaev (2006, Chapters 3 and 4) or Shiryaev (1978, Chapter 3)) lead us to formulate the following free-boundary problem for the unknown function $U_{\lambda}$ and the unknown boundary $b$ :

$$
\begin{gathered}
\left(\mathbb{L} U_{\lambda}\right)(x)=0, \quad x \in(0, b), \\
U_{\lambda}(x)>x^{2}-\lambda x, \quad x \in(0, b), \\
U_{\lambda}(x)=x^{2}-\lambda x, \quad x \in[b, \infty), \\
U_{\lambda}(0+)=0, \\
U_{\lambda}(b)=b^{2}-\lambda b \quad \text { (continuous fit), } \\
U_{\lambda}^{\prime}(b)=2 b-\lambda \quad \text { (smooth fit) }
\end{gathered}
$$

The general solution of (3.11) is $U_{\lambda}(x)=A+B x^{1+\gamma}$; the constants $A$ and $B$ and the threshold $b$ are then uniquely determined by (3.14)-(3.16). It results in $A=0, B=b^{1-\gamma}-\lambda b^{-\gamma}$ and $b=b(\lambda)=\lambda \gamma /(\gamma-1)>\lambda$, since $\gamma>1$. It is easily seen that (3.12) holds.

Now, we prove that (3.9) and (3.10) solve the optimal stopping problem (2.1). To this aim we keep using $U_{\lambda}$ for the function expressed in (2.1) and we denote by $U_{\lambda}^{\star}(x)$ the function in (3.9). By construction $U_{\lambda}^{\star}$ is $C^{2}$ on $(0, b) \cup(b, \infty)$, but $C^{1}$ at $b$. However, since the time spent by $X$ at $b$ is of Lebesgue measure 0 , Itô's formula can be applied to $U_{\lambda}^{\star}$ in its standard form:

$$
U_{\lambda}^{\star}\left(X_{t}\right)=U_{\lambda}^{\star}(x)+\int_{0}^{t}\left(\mathbb{L} U_{\lambda}^{\star}\right)\left(X_{s}\right) \mathrm{d} s+\mathcal{M}_{t},
$$

where $\mathcal{M}_{t}:=\sigma \int_{0}^{t} X_{s}^{3 / 2} U_{\lambda}^{\star^{\prime}}\left(X_{s}\right) \mathrm{d} B_{s}, t \geq 0$, is a continuous local martingale. Note that

$$
\left(\mathbb{L} U_{\lambda}^{\star}\right)(x) \leq 0, \quad x \in(0, \infty) \backslash\{b\} .
$$

This inequality holds by construction on $(0, b)$; for $x \in(b, \infty), U_{\lambda}^{\star}(x)=G_{\lambda}(x)$, so that we can easily verify that $\left(\mathbb{L} G_{\lambda}(x)\right) \leq 0$ if and only if $x \geq b / 2$. Let $\left(\tau_{n}\right)_{n \in \mathbb{N}}$ be a localizing sequence of stopping times for $\left(\mathcal{M}_{t}\right)_{t \geq 0}$. Then, from (3.12), (3.13), (3.17), and (3.18), we have

$$
\mathbb{E}_{x}\left[G_{\lambda}\left(X_{\tau \wedge \tau_{n}}\right)\right] \leq \mathbb{E}_{x}\left[U_{\lambda}^{\star}\left(X_{\tau \wedge \tau_{n}}\right)\right] \leq U_{\lambda}^{\star}(x)+\mathbb{E}_{x}\left[\mathcal{M}_{\tau \wedge \tau_{n}}\right]
$$

for any stopping time $\tau$ of $X$ such that $\mathbb{E}_{x}\left[X_{\tau}^{2}\right]<\infty$. Thus, letting $n \rightarrow \infty$ in (3.19), Fatou's lemma and the optional sampling theorem (that is, $\mathbb{E}_{x}\left[\mathcal{M}_{\tau \wedge \tau_{n}}\right]=0$ ) imply that

$$
\mathbb{E}_{x}\left[G_{\lambda}\left(X_{\tau}\right)\right] \leq U_{\lambda}^{\star}(x),
$$


which proves that $U_{\lambda}^{\star} \geq U_{\lambda}$. Repeating the same reasoning with $\tau_{b(\lambda)}^{+}$in place of $\tau$, we observe that the inequalities in (3.19) and (3.20) become equalities, so that

$$
\mathbb{E}_{x}\left[G_{\lambda}\left(X_{\tau_{b(\lambda)}^{+}}\right)\right]=U_{\lambda}^{\star}(x) \text { for any } x>0 .
$$

Equations (3.20) and (3.21) show that $U_{\lambda}^{\star}(x)=U_{\lambda}(x)$ for any $x>0$, as well as that (3.10) is optimal in (2.1). Observe that, because of the asymptotic behavior of $X$, the set in (3.10) might be empty, so that $\tau_{b(\lambda)}^{+}$is not necessarily finite valued. However, this fact does not contradict its optimality, since we can easily verify that the assumptions contained in Peskir and Shiryaev (2006, Corollary 2.9 and Remark 2.10, pp. 46-48) are satisfied.

By virtue of Theorem 2.1 and Proposition 3.1, we can determine the solutions to (2.3) and (1.1). The results are contained in the next theorem.

Theorem 3.1. Let $X$ satisfy (3.1) and (3.2) and let $\gamma:=2 k / \sigma^{2}>1$. Then, for a fixed $0<m \leq x$, the stopping time $\tau_{m}^{V}(x)$, optimal in (2.3), is expressed by

$$
\tau_{m}^{V}(x)=\inf \left\{t>0: X_{t}=m^{-1 / \gamma} x^{1+1 / \gamma}\right\}
$$

and the associated value function is given by

$$
V_{m}(x)=m^{1-1 / \gamma} x^{1+1 / \gamma}
$$

The optimal stopping time and the value function of the optimal variance stopping problem (1.1) are respectively given by (see Figure 1)

$$
\begin{gathered}
\tau^{\star}(x)=\inf \left\{t>0: X_{t}=\left(\frac{2 \gamma}{\gamma-1}\right)^{1 /(1+\gamma)} x\right\} \\
\mathbb{V}(x)=x^{2}\left[\left(\frac{2 \gamma}{\gamma-1}\right)^{(1-\gamma) /(1+\gamma)}-\left(\frac{2 \gamma}{\gamma-1}\right)^{(-2 \gamma) /(1+\gamma)}\right] .
\end{gathered}
$$

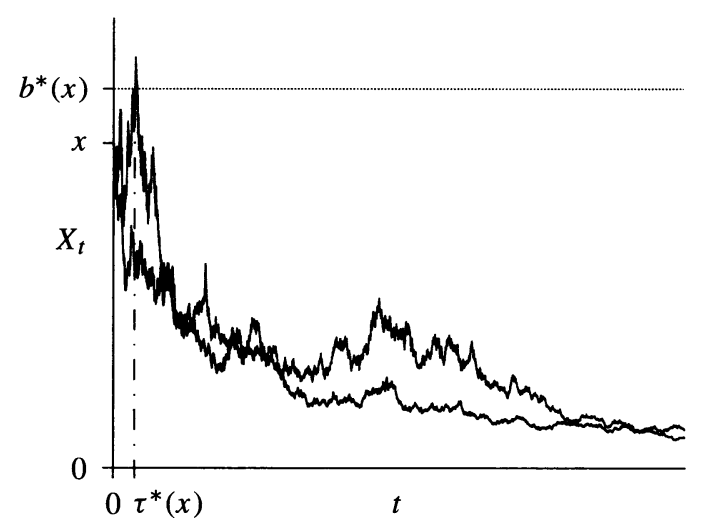

FIGURE 1: A plot of two simulated paths of $X$ from (3.1), where we set $x=0.5, k=1.5$ and $\sigma=0.8$. According to (3.23), the optimal stopping time $\tau^{\star}(x)$ for (1.1) is the first time that $X_{t}$ reaches $b^{\star}(x)=$ 0.5891 . As we can see, $\tau^{\star}(x)$ is not necessarily finite valued. 
Proof. For a given $b \geq x$, we have

$$
\mathbb{P}_{x}\left(X_{\tau_{b}^{+}}=b\right)=\mathbb{P}_{x}\left(\tau_{b}^{+}<\infty\right)=\mathbb{P}_{x}\left(\tau_{b}<\tau_{0+}\right)
$$

being $\tau_{b}^{+}$the first time that $X$ is above the threshold $b$, defined through (3.10), and $\tau_{y}$ the first hitting time of $X$ to level $y$. The equalities in (3.25) hold because $X_{t}$ converges to $0 \mathbb{P}_{x}$-a.s. as $t$ goes to $\infty$. From (3.6) and (3.25) it is easily seen that, for $x \leq b$,

$$
\begin{aligned}
& \mathbb{E}_{x}\left[X_{\tau_{b}^{+}}\right]=b \mathbb{P}_{x}\left(\tau_{b}^{+}<\infty\right)+0 \mathbb{P}_{x}\left(\tau_{b}^{+}=\infty\right)=b^{-\gamma} x^{1+\gamma}, \\
& \mathbb{E}_{x}\left[X_{\tau_{b}^{+}}^{2}\right]=b^{2} \mathbb{P}_{x}\left(\tau_{b}^{+}<\infty\right)+0 \mathbb{P}_{x}\left(\tau_{b}^{+}=\infty\right)=b^{1-\gamma} x^{1+\gamma}
\end{aligned}
$$

Observe from (3.1) that $\mathrm{X}$ is a supermartingale, since $\mu(x)=-k x^{2}<0$, implying that the set $\mathcal{C}_{x}$ of Theorem 2.1 equals $\{y>0: y \leq x\}$. So, for a fixed $0<m \leq x$, in order to meet (2.2) and using the optimality result established in Proposition 3.1 and (3.26), we set

$$
m=[b(\lambda(m, x))]^{-\gamma} x^{1+\gamma},
$$

where we recall that $b(\lambda)=\lambda \gamma /(\gamma-1)$ and $\lambda(m, x)$ is the constant that, according to Theorem 2.1, ensures the existence of an optimal stopping time for (2.3). Easy calculations imply that $\lambda(m, x)=m^{-1 / \gamma} x^{1+1 / \gamma}(\gamma-1) / \gamma$. Then, from Theorem 2.1, (3.10), and (3.28),

$$
\tau_{m}^{V}(x)=\tau_{b(\lambda(m, x))}^{+}=\inf \left\{t>0: X_{t}=m^{-1 / \gamma} x^{1+1 / \gamma}\right\}
$$

is optimal in (2.3). The equality sign in the set (3.29) follows from the fact that $m \leq x$ if and only if $b \geq x$, as we can observe from (3.28). Equation (3.22) follows from (3.27) and (3.28), or, equivalently, from $U_{\lambda(m, x)}(x)+\lambda(m, x) m$, where $U_{\lambda(m, x)}(x)$ is given through (3.9).

The optimal variance stopping problem (2.1) can now be faced as a standard optimization problem, as stated by Theorem 2.1. In particular, from $\mathcal{C}_{x}=\{y>0: y \leq x\}$, (2.5), (3.22), and (3.28), it follows that

$$
\mathbb{V}(x)=\sup _{0<m \leq x}\left(m^{1-1 / \gamma} x^{1+1 / \gamma}-m^{2}\right)=\sup _{b \geq x}\left(b^{1-\gamma} x^{1+\gamma}-b^{-2 \gamma} x^{2(1+\gamma)}\right) .
$$

Let $f(b):=b^{1-\gamma} x^{1+\gamma}-b^{-2 \gamma} x^{2(1+\gamma)}, b \geq x$, and consider its first derivative

$$
f^{\prime}(b)=(1-\gamma) b^{-\gamma} x^{1+\gamma}+2 \gamma b^{-2 \gamma-1} x^{2(1+\gamma)} \text {. }
$$

Recalling that $\gamma:=2 k / \sigma^{2}>1$, we note that the map $b \mapsto f(b)$ starts at 0 (at $b=x$ ), has an initial increase and then decreases towards 0 as $b$ goes to $\infty$. This shows that $f$ has a unique maximum point $b^{\star}(x) \in(x, \infty)$; setting $f^{\prime}(b)=0$, we obtain

$$
b^{\star}(x)=\left(\frac{2 \gamma}{\gamma-1}\right)^{1 /(1+\gamma)} x>x .
$$

According to Theorem 2.1, it thus follows that the stopping time given in (3.23) is optimal in (1.1). The value function (3.24) is finally obtained by evaluating $f\left(b^{\star}(x)\right)$.

Remark 3.1. In standard/linear optimal stopping problems the state space of the observed process $X$ can be partitioned into two sets, independently of the starting point $x$ : they are the continuation set, where the value function is strictly greater (less) than the gain (loss) function, 
and the stopping set, where the value function equals the gain or loss function, as shown for example by Proposition 3.1. The results of Pedersen (2011) and Theorem 3.1 clearly show that this situation does not hold for (1.1), whose optimal stopping boundary depends on the starting point $x$ of $X$. This is a remarkable fact that led Pedersen and Peskir (2012) to introduce the concepts of static and dynamic optimality.

In our context, we say that a stopping time $\tau_{\star}$ is statically optimal in (1.1) if there is no other stopping time $\tau$ such that

$$
\operatorname{var}_{x}\left[X_{\tau}\right]>\operatorname{var}_{x}\left[X_{\tau_{\star}}\right]
$$

It is evident that the meaning of optimal variance stopping problem illustrated at beginning of this paper is equivalent to finding a stopping time which is optimal in (1.1) in the static sense. Hence, the stopping time in (3.23) is statically optimal.

A stopping time $\tau_{\star}$ is instead dynamically optimal in (1.1) if there is no other stopping time $\tau$ such that

$$
\mathbb{P}_{x}\left(\operatorname{var}_{\tau_{\tau_{\star}}}\left[X_{\tau}\right]>0\right)>0 \text {. }
$$

This criterion basically states that the stopping time $\tau_{\star}$ is optimal in the dynamic sense if once the process $X$ is stopped at $\tau_{\star}$ and the optimal stopping problem (1.1) with the new starting point $X_{\tau_{\star}}$ is considered, there is no hope to improve the gain at a future stopping time. It is very intuitive to see that in (1.1) it is dynamically optimal not to stop at all and this is formally justified by the fact that the stopping boundary appearing in (3.23) is always greater than the initial point.

\section{Conclusions}

In this paper we analyzed a method for handling an optimal variance stopping problem and we illustrated it through a financial application. We observe that the solution method presented in Theorem 2.1 does not seem to be restricted to quadratic optimal stopping problems, like problem (1.1) or the one faced by Pedersen and Peskir (2012). Indeed, we think that the examined approach could find a more general application also in other highly nonlinear optimal stopping problems, which are certainly worthy of further investigation in future studies.

\section{Acknowledgement}

The author expresses his gratitude to the anonymous referee for precise and constructive comments that improved the presentation of the paper.

\section{References}

Ahn, D.-H. AND GaO, B. (1999). A parametric nonlinear model of term structure dynamics. Rev. Financial Studies 12, 721-762.

Aït-Sahalia, Y. (1996). Testing continuous-time models of the spot interest rate. Rev. Financial Studies 9, $385-426$.

Conley, T. G., Hansen, L. P., Luttmer, E. G. J. And Scheinkman, J. A. (1997). Short-term interest rates as subordinated diffusions. Rev. Financial Studies 10, 525-577.

Karatzas, I. And Shreve, S. E. (1988). Brownian Motion and Stochastic Calculus. Springer, New York.

Karlin, S. And Taylor, H. M. (1981). A Second Course in Stochastic Processes. Academic Press, New York.

Kutoyants, Y. A. (2004). Statistical Inference for Ergodic Diffusion Processes. Springer, London.

Pedersen, J. L. (2011). Explicit solutions to some optimal variance stopping problems. Stochastics 83, 505-518.

Pedersen, J. L. ANd Peskir, G. (2012). Optimal mean-variance selling strategies. To appear in Math. Financ. Econ.

Peskir, G. AND Shiryaev, A. (2006). Optimal Stopping and Free-Boundary Problems. Birkhäuser, Basel.

ShIRYAEV, A. N. (1978). Optimal Stopping Rules. Springer, New York. 